\title{
Study of GaN-Based Photonic Crystal Surface-Emitting Lasers (PCSELs) With AlN/GaN Distributed Bragg Reflectors
}

\author{
Shih Wei Chen, Tien Chang Lu, Member, IEEE, and Tsung Ting Kao
}

\begin{abstract}
GaN-based 2-D photonic crystal (PC) surfaceemitting lasers (PCSELs) with AIN/GaN distributed Bragg reflectors are fabricated and investigated. A clear threshold characteristic under the optical pumping at room temperature is observed at about $2.7 \mathrm{~mJ} / \mathrm{cm}^{2}$ with PC lattice constant of $234 \mathrm{~nm}$. Above the threshold, only one dominant peak appears at $401.8 \mathrm{~nm}$ with a linewidth of $0.16 \mathrm{~nm}$. The laser emission covers whole circular 2-D PC patterns of $50 \mu \mathrm{m}$ in diameter with a small divergence angle. The lasing wavelength emitted from 2-D PC lasers with different lattice constants occurs at the calculated band edges, showing different polarization angles due to the light diffracted in specific directions, corresponding exactly to $\Gamma$-, $K$-, and $M$-directions in the $K$ space. The PCSEL also shows a spontaneous emission coupling factor $\beta$ of $5 \times 10^{-3}$ and a characteristic temperature of 148 $K$. Furthermore, the coupled-wave model in 2-D hexagonal lattice is applied to distinguish the discrepancy in threshold power and the corresponding coupling coefficient. The results show that the lasing actions within $\Gamma, K$, and $M$ modes have a substantial relation between the threshold energy density and the coupling coefficient.
\end{abstract}

Index Terms-GaN, hexagonal lattice, photonic crystal (PC), surface-emitting lasers.

\section{INTRODUCTION}

D URING the past decade, photonic crystals (PCs) have drawn much attention, and have many advantages to control the light propagation and photonic bandgap. The applications of PCs nanostructure can function as good photonic devices [1]-[3]. In general, there are two types of PC lasers, which have been developed and investigated. The first one fabricates a PC defect with an optical gain surrounded by 2-D or 3-D PC mirrors to form a resonant cavity, and lasing actions arise from the resonant cavity modes with high $Q$-values and a small modal volume. This kind of microcavity laser can achieve strong Purcell effect and the low-threshold lasing [4], [5].

Another type of PC lasers can operate without any defined cavity and extrinsic mirror because Bloch waves have an intrinsic feedback mechanism near the bandgap edge. Thus,

Manuscript received October 31, 2008. First published February 24, 2009; current version published June 5, 2009. This work was supported in part by the Ministry of Education (MOE) Aim for the Top University (ATU) Program, Nano Facility Center, and in part by the National Science Council, Taipei, Taiwan, under Contract NSC95-3114-P-009-001-MY2 and Contract NSC962120-M009-006.

The authors are with the Department of Photonics and the Institute of Electro-Optical Engineering, National Chiao Tung University, Hsinchu 300, Taiwan (e-mail: macub.eo95g@nctu.edu.tw; timtclu@ faculty.nctu.edu.tw; ttkao@faculty.nctu.edu.tw).

Color versions of one or more of the figures in this paper are available online at http://ieeexplore.ieee.org.

Digital Object Identifier 10.1109/JSTQE.2008.2010877 this kind of PC laser is called as the band edge laser. To date, there have been several reports on this type of laser such as using InP, organic, and GaN material systems [6]-[9], whereas detailed analysis, such as emission modes, polarization, characteristic temperature, spontaneous coupling factor, and lasing characteristics of different band edges have not been reported.

In this study, the nitride-based band edge PC lasers with a distributed Bragg reflector (DBR) were fabricated and investigated. The PC laser structure consisted of 29 pairs of AlN/GaN DBR, n-type GaN, multiquantum wells (MQWs), and p-type GaN grown by metal-organic chemical vapor deposition system. The optical characteristics of the PC lasers with different PC lattices including the threshold energy densities, the transition behavior from spontaneous emission to stimulated emission, characteristic temperature, laser polarization, and spontaneous emission coupling factors, were obtained under the optical pumping conditions. The bottom DBR can play a role as a low refractive layer in the whole structure, leading to the confined light fields of the fundamental guided mode in the active region with large confinement factors [10]. Above the threshold energy density, the laser emission covering whole circular 2-D PC patterns is directly emitted out of the in-plane surface with a small divergence angle, forming surface-emitting laser schemes, where the name of photonic crystal surface-emitting laser (PCSEL) was thus obtained. Besides, the lasing wavelength emitted from GaN-based PC lasers with different lattice constants occurred at the calculated band edges, showing that different polarization angles indicated different lasing modes corresponding to $\Gamma, K$, and $M$ Brillouin zone boundaries [11]. Moreover, we calculated the coupled-wave model in 2-D hexagonal lattice to explain the discrepancy in threshold power with a TE-like mode by considering higher components of Bloch modes and the corresponding coupling coefficients [12]-[14]. The characteristics of large-area, small-divergence-angle, and single-mode emission from the GaN-based PCSELs show high potential in high-power blue-violet emitter applications.

\section{EXPERIMENTAl Process AND SAMPLE Structure}

The GaN-based PCSEL structure shown schematically in Fig. 1(a) was grown by a metal-organic chemical vapor deposition system on a $c$-face 2-in-diameter sapphire. Trimethylindium, trimethylgallium, trimethylaluminum, and ammonia were used as the $\mathrm{In}, \mathrm{Ga}, \mathrm{Al}$, and $\mathrm{N}$ sources, respectively. The 35-pair quarter-wave GaN/AIN DBR grown on 

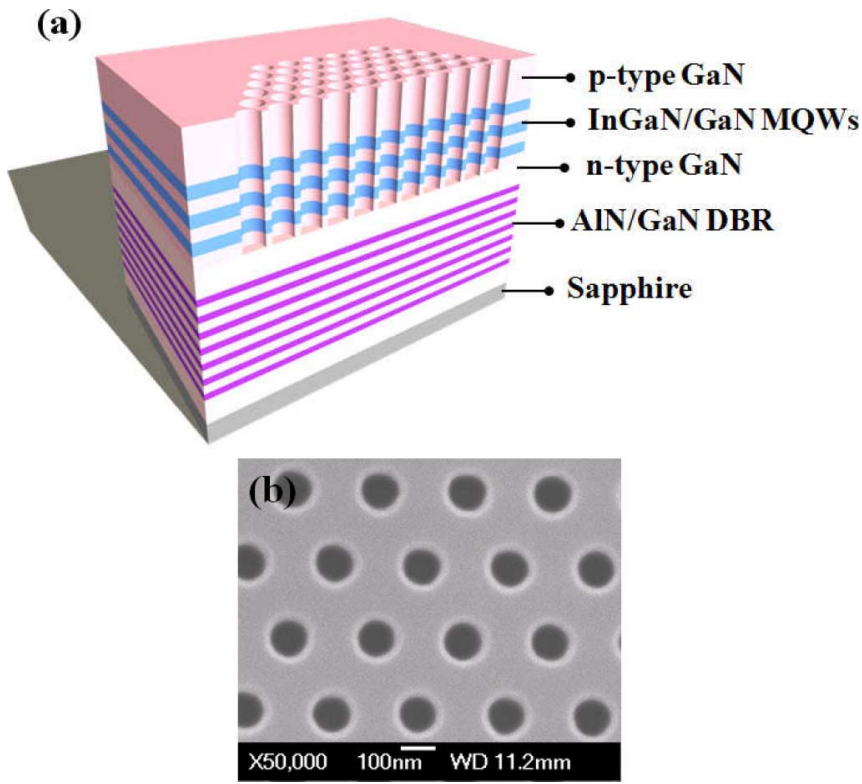

Fig. 1. (a) Schematic layer structure of GaN-based PCSELs with bottom AIN/GaN DBR. (b) Top-view SEM image of the PC structure with hexagonal lattices and circle unit cells.

a 2- $\mu$ m-thick undoped GaN buffer layer was crack-free and had a flat surface. The detailed growth parameters were reported elsewhere [15]. Then, an active region grown atop the DBR typically composed of ten $\operatorname{In}_{0.2} \mathrm{Ga}_{0.8} \mathrm{~N}$ QWs $\left(L_{W}=2.5 \mathrm{~nm}\right)$ with $\mathrm{GaN}$ barriers $\left(L_{B}=7.5 \mathrm{~nm}\right)$, and was surrounded by a 560-nm-thick Si-doped n-type GaN and a 200-nm-thick Mgdoped p-type GaN layer.

The as-grown sample was then first deposited with a hard mask consisting of an $\mathrm{SiN}_{x}$ layer of $200 \mathrm{~nm}$ by plasma-enhanced chemical vapor deposition, followed by a soft mask consisting of a polymethylmethacrylate (PMMA) layer of $150 \mathrm{~nm}$. Using electron beam lithography, we defined on the soft mask a hexagonal PC pattern with the lattice constant $a$ ranging from 190 to $300 \mathrm{~nm}$ and the circular hole diameter $r$ chosen such that $r / a$ ranged from 0.18 to 0.3 . The whole PC pattern was of circular and hexagonal shapes with a diameter of $50 \mu \mathrm{m}$. Then, the PC pattern on soft mask was transferred to $\mathrm{SiN}_{x}$ film by inductively coupled plasma-reactive ion etching (ICP-RIE). After the PMMA layer was removed by acetone, we used ICP-RIE to etch down the as-grown sample to about $400 \mathrm{~nm}$ deep. The etching process penetrated the MQWs active regions and created the PC patterns in the nitride layers. Finally, the $\operatorname{SiN}_{x}$ hard mask was removed by buffered oxide etch dipping. The top view of the hexagonal PC pattern on the GaN-based structure thus created is shown in Fig. 1(b).

\section{MEASUREMENT System AND CHARACTERISTICS OF PCSELS}

The typical photoluminescence (PL) spectrum of the PC surface emission structure had a peak centered at a wavelength of $415 \mathrm{~nm}$ with a linewidth of about $25 \mathrm{~nm}$. At normal incidence at room temperature, the DBR showed the highest reflectivity of

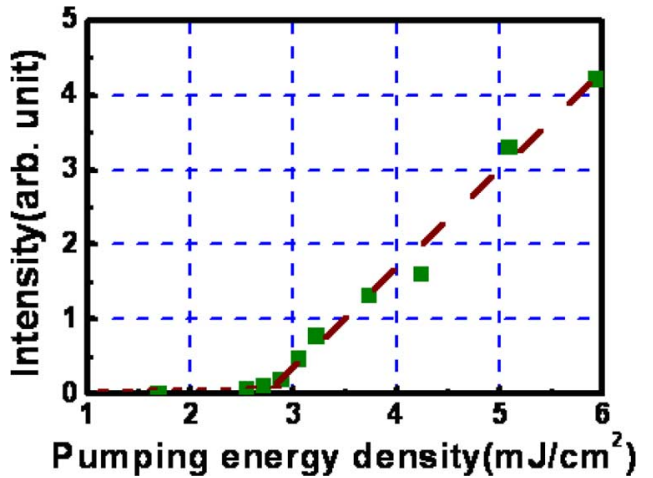

Fig. 2. Measured output intensity versus input excitation energy density from the GaN-based PCSELs with bottom AIN/GaN DBRs at room temperature.

$99 \%$ at the center wavelength of $415 \mathrm{~nm}$, with a stopband width of about $30 \mathrm{~nm}$, measured by an $n k \mathrm{UV}$-Vis spectrometer.

The emission spectra of the GaN-based PC surface emission structure were measured by using a microscopy system (WITec, Alpha SNOM). The optical pumping was performed by a Nd: $\mathrm{YVO}_{4} 355 \mathrm{~nm}$ pulsed laser with a pulsewidth of about $0.5 \mathrm{~ns}$ at a repetition rate of $1 \mathrm{kHz}$. The pumping laser beam, with a spot size of $50 \mu \mathrm{m}$, was normally incident onto the sample surface and covered the whole PC pattern area. A $15 \times$ objective lens with a numerical aperture of 0.32 was placed normal to the sample to collect the light emission from the top of the PC pattern. The light was then collected by a fiber with a $600 \mu \mathrm{m}$ core and coupled into a spectrometer with a charge-coupled device (CCD). The spectral resolution is about $0.1 \mathrm{~nm}$ for spectral output measurement. The GaN-based PC surface-emitting lasers were placed in a cryogenics-controlled chamber for obtaining the characteristic temperature. The temperature of the chamber can be controlled from room temperature, $300 \mathrm{~K}$, down to $100 \mathrm{~K}$ using the liquid nitrogen.

Fig. 2 shows the laser intensity as a function of the pumping energy at room temperature condition from the sample with PC lattice constant of $234 \mathrm{~nm}$. The clear evidence of threshold condition occurred at the pumping energy $\left(E_{\mathrm{th}}\right)$ of $165 \mathrm{~nJ}$ corresponding to an energy density of $2.7 \mathrm{~mJ} / \mathrm{cm}^{2}$. The output laser intensity from the sample increased linearly and abruptly with the pumping energy density beyond the threshold.

Fig. 3 shows the excitation-energy-density-dependent emission spectra. These spectra clearly show the transition behavior from spontaneous emission to stimulated emission with a single dominant peak. Above the threshold, we can observe only one dominant peak wavelength of $401.8 \mathrm{~nm}$ with a full width at half maximum (FWHM) of $0.16 \mathrm{~nm}$ limited by our measurement resolution.

The lasing area of the GaN-based PCSEL obtained by a CCD camera is relatively large that covers almost whole area of the PC pattern with only one dominant lasing wavelength. The measured FWHM of laser emission divergence angle is smaller than $5^{\circ}$, indicating that the surface emission is almost normal to the PC surface. It is interesting to note that the threshold power density of GaN-based 2-D surface-emitting PC laser is in the same order of or even better than the threshold for GaN-based 


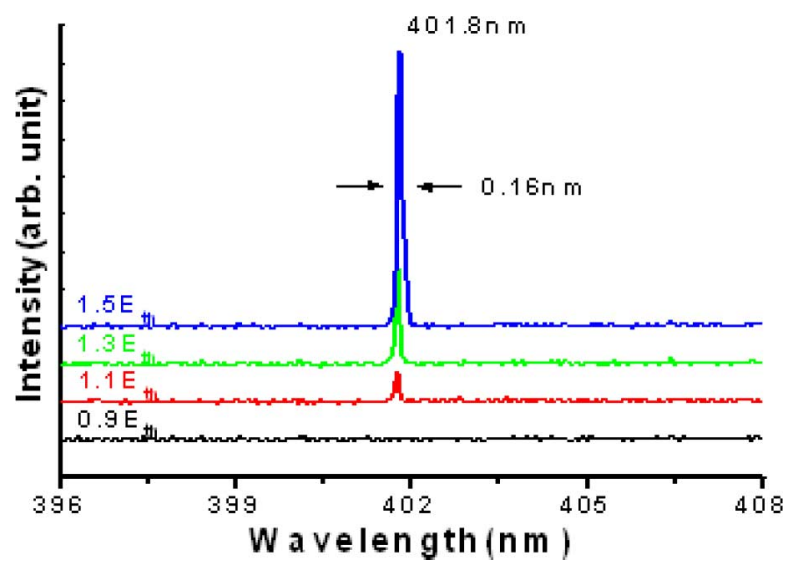

Fig. 3. Emission spectra under varied excitation energy density from the GaNbased PCSELs with bottom AIN/GaN DBRs at room temperature.
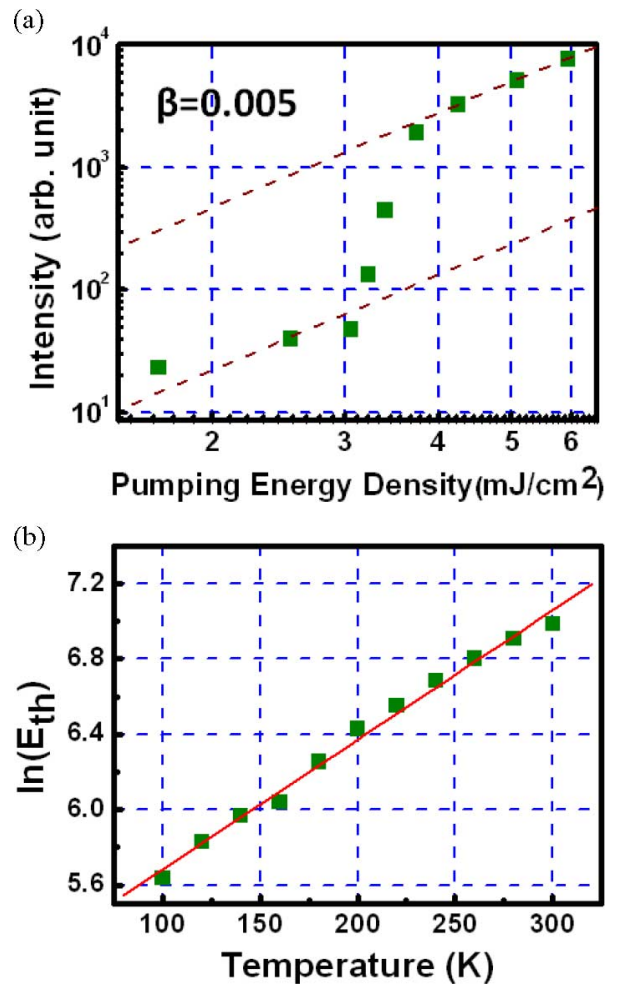

Fig. 4. (a) Laser emission intensity versus pumping energy in a logarithmic scale. The $\beta$-value estimated from the difference between the two dashed lines is about $5 \times 10^{-3}$. (b) Temperature dependence of the lasing threshold pumping energy $\left(E_{\mathrm{th}}\right)$.

vertical-cavity surface-emitting lasers (VCSELs) that we demonstrated recently [16]. Unlike the small emission spots observed in the GaN-based VCSELs, the large-area emission in PCSELs has great potential in applications requiring highpower-output operation.

To understand the spontaneous coupling factor $\beta$ of the GaNbased PCSELs, we further replotted the laser emission intensity versed pumping energy from Fig. 2 in logarithmic scale, as shown in Fig. 4(a), and then calculated the difference between the heights of the emission intensities before and after the threshold, corresponding roughly to the value of $\beta$.

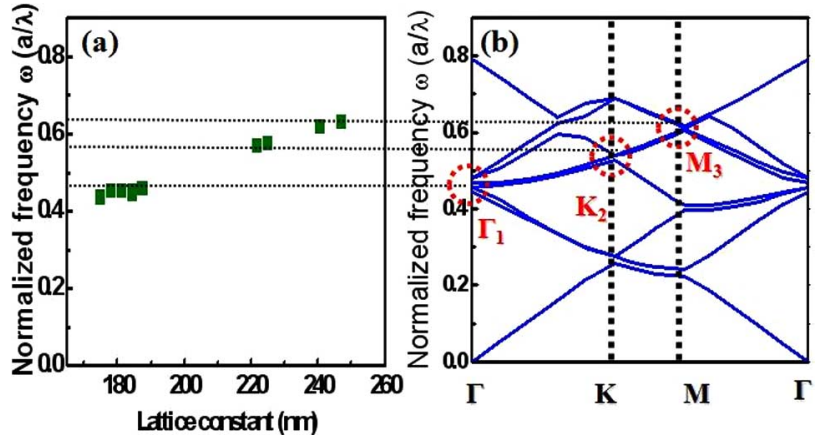

Fig. 5. (a) Normalized frequency verses PC lattice constant. The solid square points are the lasing wavelengths from the different PC structures. (b) Calculated band diagram of the 2-D hexagonal lattice structure. The dotted lines are guides for band edges.

The $\beta$-value of our PC surface- emitting laser was estimated to be about $5 \times 10^{-3}$. Interestingly, this value is smaller than the GaN-based VCSELs [16], which could be due to the lower quality factor in this GaN-based PC structure. However, the $\beta$-factor is still larger than the typical edge emitting lasers (normally about $10^{-5}$ ), indicating the enhancement of the spontaneous emission into a lasing mode provided by the high distributed feedback effect in GaN-based PCSELs.

Fig. 4(b) shows the seminatural logarithm plots of the dependence of the threshold pumping energy $\left[\ln \left(E_{\mathrm{th}}\right)\right]$ on the operation temperature $\left(T_{0}\right)$. The threshold energy gradually increased as the operation temperature rose from 100 to $300 \mathrm{~K}$. The relationship between the threshold energy and the operation temperature could be characterized by the equation $E_{\mathrm{th}}=E_{0} e^{T / T_{0}}$, where $T_{0}$ is the characteristic temperature and $E_{0}$ is a constant. Therefore, we obtained a characteristic temperature of about $148 \mathrm{~K}$ by linear fitting of the experimental data, which is close to the value reported for GaN-based edge emitting lasers [17].

\section{2-D Plane-WAVE ExPANSION CALCUlation}

The single-mode lasing phenomenon occurs only in the area with PC patterns. The normalized frequency (lattice constant over wavelength, $a / \lambda$ ) for the lasing wavelength emitted from our PC lasers with different lattice constants were plotted, as shown in Fig. 5(a). All the PC lasers have lasing peaks in a range from 401 to $425 \mathrm{~nm}$. It can be seen that the normalized lasing frequency (dotted points in the figure) increased with the lattice constant in a discontinuous and step-like fashion. To calculate the band diagram of the hexagonal PC patterns in this structure, we employed the plane-wave expansion method in two dimensions with an effective index approach that took into account the effects of partial modal overlap of electromagnetic fields with the PC structures [9]. As a starting point, the overlapped ratio of light confined within the 2-D PC structure to light extended in the entire device, $\Gamma_{g}$, and the effective refractive index of the entire device $n_{\text {eff }}$ were first estimated by using the transfer matrix method. The calculation showed that the lowest order guided mode had the highest confinement factor for both PC and MQW regions, and the $\Gamma_{g}$ and $n_{\text {eff }}$ were estimated to be 0.563 and 2.495 , respectively. Then, we 


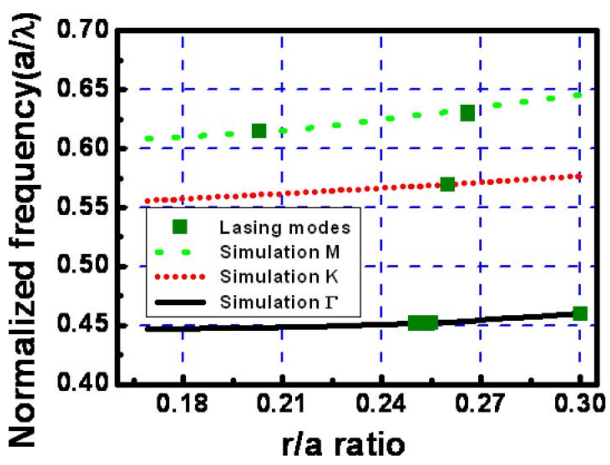

Fig. 6. Normalized frequency verses $r / a$ ratios. The solid (black), dotted (red), and dashed (green) lines represent the simulation results of $\Gamma, K$, and $M$ lasing groups by plane-wave expansion method. The square points present the experiment results mapped and compared with the simulation results.

determined the effective dielectric constants of the two materials in the unit cell, $\varepsilon_{a}$ and $\varepsilon_{b}$, using $n_{\text {eff }}^{2}=f \varepsilon_{a}+(1-f) \varepsilon_{b}$ and $\Delta \varepsilon=\varepsilon_{b}-\varepsilon_{a}=\Gamma_{g}\left(\varepsilon_{\text {mat }}-\varepsilon_{\text {air }}\right)$, where $f=2 \pi r^{2} /\left(a^{2} \sqrt{3}\right)$ is a filling factor, and $\varepsilon_{\mathrm{mat}}$ and $\varepsilon_{\text {air }}$ are dielectric constants of $\mathrm{GaN}$ $\left(=2.5^{2}\right)$ and air $\left(=1^{2}\right)$, respectively. The values of $\varepsilon_{a}(4.11)$ and $\varepsilon_{b}$ (7.07) thus obtained were then put into the calculation of the band diagram for the 2-D hexagonal lattice structure with $r / a=0.25$.

Fig. 5(b) shows the calculated band diagram of the 2-D hexagonal lattice structure for TE mode. It can be expected that the lasing occurs at special points such as at Brillouin zone boundary near the band edges, because the Bragg condition is satisfied and the density of states is higher at these band edges [9]. At these lasing points, wave can propagate in different directions and couple with each other. The dotted lines are guides for band edges calculated in Fig. 5(b) and extended horizontally to Fig. 5(a) with the same normalized frequency. It can be seen that different groups of the normalized frequency observed in the PC samples with different lattice constants occur exactly at band edges such as $\Gamma, M$, and $K$ points (or more specifically labeled as $\Gamma 1, K 2, M 3)$, indicating that the laser operation was provided by multidirectional distributed feedback in the 2-D PC structure [7]. The characteristics of $\Gamma, M$, and $K$ points lasing can be further identified by the polarization angle of the output emission. Note that the output intensity is higher when some of the lasing frequencies are in the stopband of DBR, which could be due to the fact that the bottom DBR here can be treated as a high reflectivity reflector, facilitating top emission efficiency.

Different lasing frequencies were measured from different PC lattice structures. The normalized frequencies as a function of $r / a$ ratio were plotted as square points in Fig. 6. The solid (black), dotted (red), and dashed (green) lines are the calculated band edge frequencies at the $\Gamma, K$, and $M$ Brillouin zone boundaries as a function of $r / a$ ratio, which were in accordance with the measured results.

\section{LASing POlarization Direction In REAL SPACE CORRESPONDING TO $K$-SPACE}

The measured polarization curves for different band edge lasers grouped into $\Gamma$ (red: circle points and solid line), $K$ (green:
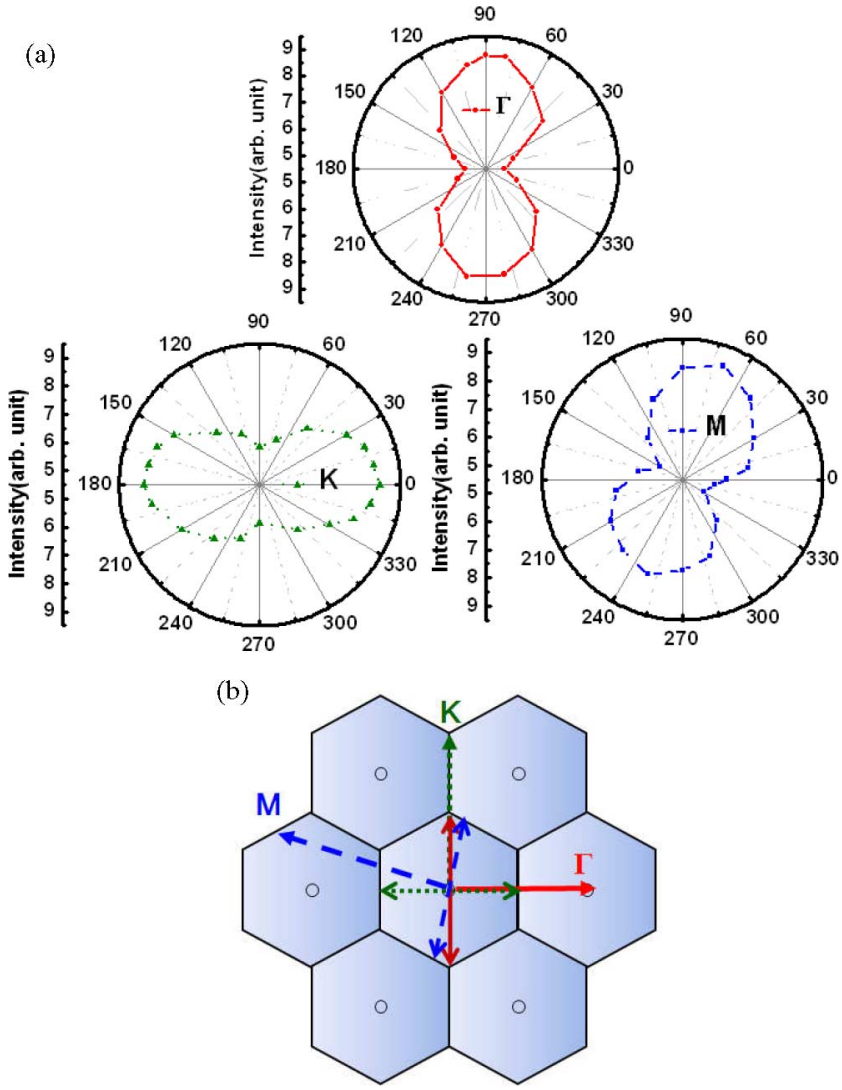

Fig. 7. (a) Measured polarization curves for different band edge lasers grouped into $\Gamma$ (red: circle points and solid line), $K$ (green: triangle points and dotted line), and $M$ (blue: square points and dashed line) boundaries calculated by the plane-wave expansion method. (b) Main polarization directions obtained in (a) and their corresponding diffracted laser beams, which are normal to the polarization directions in a $K$-space map corresponding to our hexagonal PC lattice.

triangle points and dotted line), and $M$ (blue: square points and dashed line) boundaries calculated by the plane-wave expansion method are shown in Fig. 7(a), and the degree of polarization from the emission defined as $\left(I_{\max }-I_{\min }\right) /\left(I_{\max }+I_{\min }\right)$ was somehow around $50 \%$. The polarization angles from the emissions of devices with different normalized frequencies grouped into $\Gamma, K$, or $M$ band edge lasers were different. Since the PC lattices provide the optical feedback, which is the origin of the band edge laser operation, the direction and the polarization of the laser light will strictly follow the PC lattice vectors. The symmetric feedback directions provided by the 2-D lattice vectors could result in a relatively low degree of polarization when the measurement of the polarization is from the top of the device [18]. As a result, it could be rather difficult to distinguish the specific polarization directions in PCSELs whenever they are categorized as $\Gamma, K$, or $M$ band edge lasers. However, the feedback beams could not be equally diffracted by PC lattices probably due to some disorders or imperfections in the fabricated PC structure. This will result in some beams diffracted in specific directions having higher intensity. The ideally symmetric polarization directions will also be broken. The preferred polarization directions for different band edge lasers should follow specific directions. The main polarization directions and 


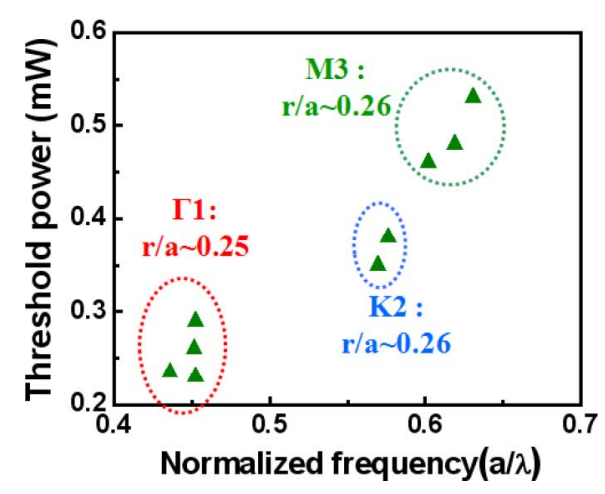

Fig. 8. Different threshold power versus normalized frequency.

the main diffracted laser beams, which are normal to the main polarization directions, can be drawn in a $K$-space map corresponding to our hexagonal PC lattice, as shown in Fig. 7(b). These main diffracted laser beams, shown as dashed lines in Fig. 7 (b), point exactly to the $\Gamma$ (solid red line), $K$ (dotted green line), and $M$ (dashed blue line) boundaries. The distinct polarization directions provide solid evidence that the lasing actions of our PC laser indeed originate from different band edges.

\section{Coupled-WaVe Model Calculation}

From Figs. 5 and 7, we can obtain the laser emission that precisely occurs at three different band edge frequencies ( $\Gamma 1, K 2, M 3)$ with different threshold pumping powers. As shown in Fig. 8, we are able to classify the lasing actions into three groups, where lasing within $\Gamma 1$ mode has the lowest threshold power while lasing within M3 mode has the highest threshold power. Such results could be attributed to the different coupling effects at different band edges, which can be explained by coupled-wave model calculation.

To simplify the calculation, the 2-D PC structure investigated here consists of an infinite hexagonal lattice with circular air holes in the $x$ - and $y$-directions. The corresponding 2-D $K$-space is shown in Fig. 9. The structure is also assumed to be uniform in the $z$-direction. In addition, the gain effects are neglected here during the calculation to extract the pure diffraction effects. While considering the infinite structure, the scalar wave equation for the magnetic field $H_{z}$ in the TE mode can be written as [12]-[14]

$$
\frac{\partial^{2} H_{z}}{\partial x^{2}}+\frac{\partial^{2} H_{z}}{\partial y^{2}}+k^{2} H_{z}=0
$$

where

$$
k^{2}=\beta^{2}+2 j \alpha \beta+2 \beta \sum_{G \neq 0} k(G) \exp [j(G \cdot r)] .
$$

And the magnetic field can be described by the Bloch mode when considering the $\Gamma_{1}$ band edge, as shown in Fig. 9

$$
H_{z}(r)=\sum_{G} h_{G} \exp [j(k+G) r]
$$

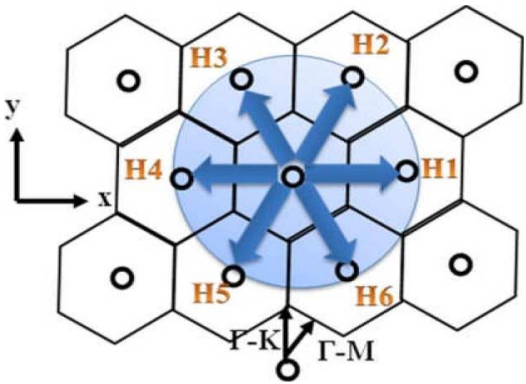

Fig. 9. Schematic diagram of six propagation waves in hexagonal lattice for $\Gamma 1$ band edge.

where

$$
\begin{aligned}
H_{z}= & H_{1}(x, y) e^{-i \beta_{0} \hat{x}}+H_{2} e^{-i \beta_{0}((1 / 2) \hat{x}+(\sqrt{3} / 2) \hat{y})} \\
& +H_{3} e^{-i \beta_{0}((-1 / 2) \hat{x}+(\sqrt{3} / 2) \hat{y})}+H_{4} e^{i \beta_{0} \hat{x}} \\
& +H_{5} e^{-i \beta_{0}((-1 / 2) \hat{x}-(\sqrt{3} / 2) \hat{y})}+H_{6} e^{-i \beta_{0}((1 / 2) \hat{x}-(\sqrt{3} / 2) \hat{y})} .
\end{aligned}
$$

Substituting (4) and (2) into (1), and comparing the equal exponential terms, we can obtain six wave equations

$$
\begin{aligned}
&-\frac{\partial}{\partial x} H_{1}+(-\alpha-i \delta) H_{1} \\
&=-i \frac{\kappa_{1}}{2}\left(H_{2}+H_{6}\right)+i \frac{\kappa_{2}}{2}\left(H_{3}+H_{5}\right)+i \kappa_{3} H_{4} \\
&-\frac{1}{2} \frac{\partial}{\partial x} H_{2}-\frac{\sqrt{3}}{2} \frac{\partial}{\partial y} H_{2}+(-\alpha-i \delta) H_{2} \\
&=-i \frac{\kappa_{1}}{2}\left(H_{1}+H_{3}\right)+i \frac{\kappa_{2}}{2}\left(H_{4}+H_{6}\right)+i \kappa_{3} H_{5} \\
&-\frac{1}{2} \frac{\partial}{\partial x} H_{3}-\frac{\sqrt{3}}{2} \frac{\partial}{\partial y} H_{3}+(-\alpha-i \delta) H_{3} \\
&=-i \frac{\kappa_{1}}{2}\left(H_{2}+H_{4}\right)+i \frac{\kappa_{2}}{2}\left(H_{1}+H_{5}\right)+i \kappa_{3} H_{6} \\
& \frac{\partial}{\partial x} H_{4}+(-\alpha-i \delta) H_{4} \\
&=-i \frac{\kappa_{1}}{2}\left(H_{3}+H_{5}\right)+i \frac{\kappa_{2}}{2}\left(H_{2}+H_{6}\right)+i \kappa_{3} H_{1}
\end{aligned}
$$

$$
\begin{aligned}
\frac{1}{2} \frac{\partial}{\partial x} H_{5} & +\frac{\sqrt{3}}{2} \frac{\partial}{\partial y} H_{5}+(-\alpha-i \delta) H_{5} \\
& =-i \frac{\kappa_{1}}{2}\left(H_{4}+H_{6}\right)+i \frac{\kappa_{2}}{2}\left(H_{1}+H_{3}\right)+i \kappa_{3} H_{2}
\end{aligned}
$$

$$
\begin{aligned}
-\frac{1}{2} \frac{\partial}{\partial x} H_{6} & +\frac{\sqrt{3}}{2} \frac{\partial}{\partial y} H_{6}+(-\alpha-i \delta) H_{6} \\
& =-i \frac{\kappa_{1}}{2}\left(H_{1}+H_{5}\right)+i \frac{\kappa_{2}}{2}\left(H_{2}+H_{4}\right)+i \kappa_{3} H_{3}
\end{aligned}
$$

where $H_{1}, H_{2}, H_{3}, H_{4}, H_{5}$, and $H_{6}$ express the envelope magnetic field distributions of individual lightwaves propagating in the six equivalent $\Gamma$ - and $M$-directions: $0^{\circ},+60^{\circ},+120^{\circ},+180^{\circ},+240^{\circ}$, and $+300^{\circ}$ with respect to the $x$-axis. $\kappa_{1}, \kappa_{2}$, and $\kappa_{3}$ are the coupling coefficients between lightwaves propagating at $60^{\circ}$ to each other $\left(H_{1}\right.$ and $H_{2}, H_{2}$ 
and $H_{3}$, etc.), at $120^{\circ}$ ( $H_{1}$ and $H_{3}, H_{2}$ and $H_{4}$, etc.), and at $180^{\circ}$ ( $H_{1}$ and $H_{4}, H_{2}$ and $H_{5}$, etc.), respectively; $\delta$ is the deviation of the wave number $\beta$ (expressed as $2 \pi \nu / c$, where $\nu$ is the frequency and $c$ is the velocity of light) from the fundamental propagation constant $\beta_{0}$ (equal to $4 \pi / \sqrt{3} a$, where $a$ is the lattice constant) for each cavity mode, and expressed as $\delta=\left(\beta^{2}-\beta_{0}^{2}\right) / 2 \beta_{0}$; and $\alpha$ is the corresponding threshold gain.

By solving (5a)-(5e), an eigenfrequency $\nu$ for each band edge mode and the corresponding threshold gain $\alpha$ for a given set of coupling coefficients, $\kappa_{1}, \kappa_{2}$, and $\kappa_{3}$, can be obtained. When only the eigenfrequencies are concerned, the derivation terms and the threshold gain $\alpha$ in (5a)-(5e) can be set to zero, and the individual eigenfrequencies can then be derived as follows:

$$
\begin{aligned}
\nu_{1} & =\frac{c}{2 \pi n_{\mathrm{eff}}}\left(\beta_{0}-\kappa_{1}-\kappa_{2}+\kappa_{3}\right) \\
\nu_{2} & =\frac{c}{2 \pi n_{\mathrm{eff}}}\left(\beta_{0}-\frac{1}{2} \kappa_{1}+\frac{1}{2} \kappa_{2}-\kappa_{3}\right) \\
\nu_{3} & =\frac{c}{2 \pi n_{\mathrm{eff}}}\left(\beta_{0}+\kappa_{1}-\kappa_{2}-\kappa_{3}\right) \\
\nu_{4} & =\frac{c}{2 \pi n_{\mathrm{eff}}}\left(\beta_{0}+\frac{1}{2} \kappa_{1}+\frac{1}{2} \kappa_{2}+\kappa_{3}\right)
\end{aligned}
$$

where $c$ is the velocity of a photon in vacuum and $n_{\text {eff }}$ is the effective refractive index of the device structure. There are four eigen-mode frequencies, $\nu_{1}-\nu_{4}$, which correspond to the four band edges, including two degenerate modes with $\nu_{2}$ and $\nu_{4}$. Once the frequency of the eigen mode at the individual band edges is obtained, the coupling coefficients $\kappa_{1}, \kappa_{2}$, and $\kappa_{3}$ can be derived from (6a)-(6d) as follows:

$$
\begin{aligned}
\kappa_{1} & =\frac{-\nu_{1}-\nu_{2}+\nu_{3}+\nu_{4}}{\nu_{1}+2 \nu_{2}+\nu_{3}+2 \nu_{4}} 2 \beta_{0} \\
\kappa_{2}= & \frac{-\nu_{1}+\nu_{2}-\nu_{3}+\nu_{4}}{\nu_{1}+2 \nu_{2}+\nu_{3}+2 \nu_{4}} 2 \beta_{0} \\
\kappa_{3} & =\frac{\nu_{1}-2 \nu_{2}-\nu_{3}+2 \nu_{4}}{\nu_{1}+2 \nu_{2}+\nu_{3}+2 \nu_{4}} 2 \beta_{0} .
\end{aligned}
$$

By comparing the value of coupling coefficients $\kappa_{1}, \kappa_{2}$, and $\kappa_{3}$ based on actual device parameters, we can determine which kind of distributed feedback mechanism provide the major significant contribution to support the lasing oscillation at $\Gamma_{1}$ band edge.

Similarly, we use the same calculation method to solve coupling coefficients for $K 2$ and $M 3$ operation modes. We can obtain two individual eigen-mode frequencies for $K 2$ and four eigen-mode frequencies for $M 3$, listed as follows.

1) $K 2$ [shown in Fig. 10(a)]: There are two eigen-mode frequencies with only one coupling angle at $120^{\circ}$ for one coupling coefficient

$$
\kappa=\frac{\nu_{2}-\nu_{1}}{\nu_{1}+2 \nu_{2}} 2 \beta_{0}
$$

2) $M 3$ [shown Fig. 10(b)]: There are four eigen-mode frequencies with three coupling angles, i.e., $82^{\circ}, 98^{\circ}$, and $180^{\circ}$, for coupling coefficients $\kappa_{1}, \kappa_{2}$, and $\kappa_{3}$

$$
\kappa_{1}=\frac{\nu_{1}-\nu_{2}-\nu_{3}+\nu_{4}}{\nu_{1}+\nu_{2}+\nu_{3}+\nu_{4}} \beta_{0}
$$
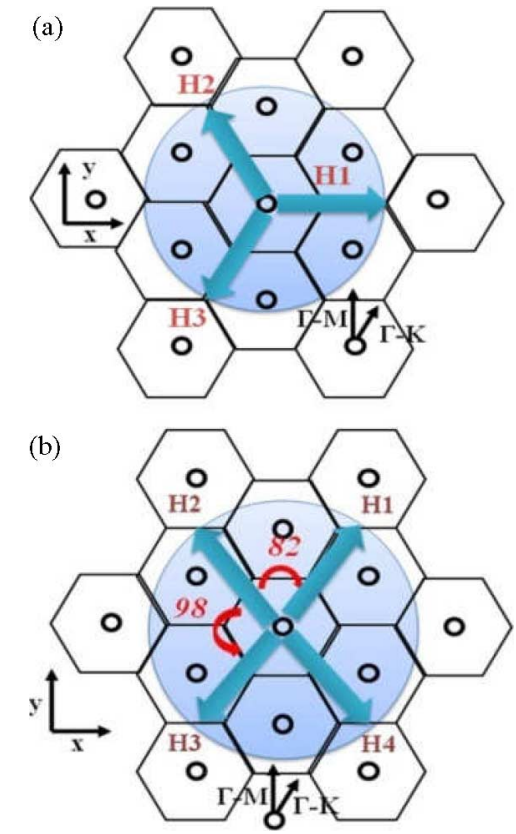

Fig. 10. Schematic diagram of propagation waves in hexagonal lattice for (a) $K 2$ band edge and (b) $M 3$ band edge.

$$
\begin{aligned}
& \kappa_{2}=\frac{-\nu_{1}+\nu_{2}-\nu_{3}+\nu_{4}}{\nu_{1}+\nu_{2}+\nu_{3}+\nu_{4}} \beta_{0} \\
& \kappa_{3}=\frac{-\nu_{1}-\nu_{2}+\nu_{3}+\nu_{4}}{\nu_{1}+\nu_{2}+\nu_{3}+\nu_{4}} \beta_{0} .
\end{aligned}
$$

The coupling coefficient of each PCSEL could then be evaluated by using the band edge frequencies, as shown in Fig. 5(b). First, for $\Gamma 1$ mode, substituting the normalized frequencies, 0.4482, 0.4595, 0.4704, and 0.4930, with the PC lattice constant of about $180 \mathrm{~nm}$ into the expressions (7a)-(7c), we obtain the coupling coefficients $\kappa_{1} \sim 17480 \mathrm{~cm}^{-1}, \kappa_{2} \sim$ $11240 \mathrm{~cm}^{-1}$, and $\kappa_{3} \sim 11268 \mathrm{~cm}^{-1}$. The largest coupling coefficient $\kappa_{1}$ shows that the lightwaves propagating at $60^{\circ}$ to each other dominate the coupling effect.

Similarly, for $K 2$ mode, substituting the normalized frequencies 0.5326 and 0.5413 with the PC lattice constant of about $220 \mathrm{~nm}$ into (8), we obtained the coupling coefficient $\kappa \sim 4089 \mathrm{~cm}^{-1}$.

For $M 3$ mode, substituting the normalized frequencies, $0.6089,0.6094,0.6141$, and 0.6234 , with the PC lattice constant of about $247 \mathrm{~nm}$ into (9a)-(9c), we obtain the coupling coefficient $\kappa_{1} \sim 1241 \mathrm{~cm}^{-1}, \kappa_{2} \sim 1356 \mathrm{~cm}^{-1}$, and $\kappa_{3} \sim 2683 \mathrm{~cm}^{-1}$. The largest coupling coefficient $\kappa_{3}$ shows that greatest feedback effect is similar to that of a conventional in-plane distributed feedback laser. Moreover, it is interesting to note that the lasing action within $\Gamma 1$ mode shows the lowest threshold power in Fig. 8, which could be attributed to the largest coupling coefficient. On the other hand, lasing within M3 mode has the highest threshold power due to the lowest coupling coefficient.

\section{CONCLUSION}

We have successfully fabricated a series of GaN-based PCSELs-incorporated AlN/GaN bottom DBRs with different 
lattice constants. Under the optical pumping at room temperature, a clear threshold characteristic was observed with a threshold energy density of about $2.7 \mathrm{~mJ} / \mathrm{cm}^{2}$ for the PC lattice of 234 $\mathrm{nm}$. Above the threshold, only one dominant peak was observed at $401.8 \mathrm{~nm}$ with a linewidth of about $0.16 \mathrm{~nm}$. Compared with photonic band diagram, and the relation between the PC lattice constants and normalized frequencies, the characteristics of $\Gamma, M$, and $K$ points lasing can be further identified by the polarization angle of the output emission. The PCSEL also shows a spontaneous emission coupling factor $\beta$ of about $5 \times 10^{-3}$ and a characteristic temperature of about $148 \mathrm{~K}$. Furthermore, the coupled-wave model in 2-D hexagonal lattice was applied to explain the discrepancy in threshold power by considering higher components of Bloch modes and the corresponding coupling coefficients. It was demonstrated that the lasing action within $\Gamma 1$ mode that had the lowest threshold power corresponded to the largest coupling coefficient. On the contrary, the highest threshold energy power occurring within M3 mode corresponded to the lowest coupling coefficient.

\section{ACKNOWLEDGMENT}

The authors would like to gratefully acknowledge S. C. Wang and H. C. Kuo from the National Chiao Tung University for their fruitful suggestion.

\section{REFERENCES}

[1] E. Yablonovitch, "Inhibited spontaneous emission in solid-state physics and electronics," Phys. Rev. Lett., vol. 58, pp. 2059-2062, Dec. 1987.

[2] S. John, "Strong localization of photons in certain disordered dielectric superlattices," Phys. Rev. Lett., vol. 58, pp. 2486-2489, Mar. 1987.

[3] J. D. Joannopoulos, P. R. Villeneuve, and S. Fan, "Photonic crystals: Putting a new twist on light," Nature, vol. 386, pp. 143-149, Mar. 1997.

[4] O. Painter, R. K. Lee, A. Scherer, A. Yariv, J. D. O'Brien, P. D. Dapkus, and I. Kim, "Two-dimensional photonic band-gap defect mode laser," Science, vol. 284, pp. 1819-1821, Jun. 1999.

[5] H. G. Park, S. H. Kim, S. H. Kwon, Y. G. Ju, J. K. Yang, J. H. Baek, S. B. Kim, and Y. H. Lee, "Electrically driven single-cell photonic crystal laser," Science, vol. 305, pp. 1444-1447, Sep. 2005.

[6] S. H. Kwon, H. Y. Ryu, G. H. Kim, Y. H. Lee, and S. B. Kim, "Photonic bandedge lasers in two-dimensional square-lattice photonic crystal slabs," Appl. Phys. Lett., vol. 83, pp. 3870-3872, Nov. 2003.

[7] M. Notomi, H. Suzuki, and T. Tamamura, "Directional lasing oscillation of two-dimensional organic photonic crystal lasers at several photonic band gaps," Appl. Phys. Lett., vol. 78, pp. 1325-1327, Mar. 2001.

[8] H. Matsubara, S. Yoshimoto, H. Saito, Y. Jianglin, Y. Tanaka, and S. Noda, "GaN photonic-crystal surface-emitting laser at blue-violet wavelengths," Science, vol. 319, pp. 445-447, Jan. 2008.

[9] M. Imada, A. Chutinan, S. Noda, and M. Mochizuki, "Multidirectionally distributed feedback photonic crystal lasers," Phys. Rev. B, vol. 65, pp. 195306-1-195306-8, Apr. 2002.

[10] T. C. Lu, S. W. Chen, L. F. Lin, T. T. Kao, C. C. Kao, P. C. Yu, H. C. Kuo, S. C. Wang, and S. H. Fan, "GaN-based two-dimensional surface-emitting photonic crystal lasers with AlN/GaN distributed Bragg reflector," Appl. Phys. Lett., vol. 92, pp. 011129-1-011129-3, Jan. 2008.

[11] T. C. Lu, S. W. Chen, T. T. Kao, and T. W. Liu, "Characteristics of GaNbased photonic crystal surface emitting lasers," Appl. Phys. Lett., vol. 93, pp. 111111-1-111111-3, Sep. 2008.

[12] Plihal and A. A. Maradudin, "Photonic band structure of two-dimensional systems: The triangular lattice," Phys. Rev. B, vol. 44, pp. 8565-8571, Feb. 1991.

[13] K. Sakai, J. Yue, and S. Noda, "Coupled-wave model for triangular-lattice photonic crystal with transverse electric polarization," Opt. Exp., vol. 16, no. 9, pp. 6033-6040, Apr. 2008.

[14] H. Kogelnik, "Coupled wave theory for thick hologram grating," Bell Syst. Tech. J., vol. 48, pp. 2909-2947, Nov. 1969.
[15] G. S. Huang, T. C. Lu, H. H. Yao, H. C. Kuo, S. C. Wang, C.-W. Lin, and L. Chang, "Crack-free GaN/AlN distributed Bragg reflectors incorporated with GaN/AlN superlattices grown by metalorganic chemical vapor deposition," Appl. Phys. Lett., vol. 88, pp. 061904-1-061904-3, Feb. 2006.

[16] S.-C. Wang, T.-C. Lu, C.-C. Kao, J.-T. Chu, G.-S. Huang, H.-C. Kuo, S.-W. Chen, T.-T. Kao, J.-R. Chen, and L.-F. Lin, "Optically pumped GaN-based vertical cavity surface emitting lasers: Technology and characteristics," Jpn. J. Appl. Phys., vol. 46, pp. 5397-5407, 2007.

[17] C. Skierbiszewski, P. Perlin, I. Grzegory, Z. R. Wasilewski, M. Siekacz, A. Feduniewicz, P. Wisniewski, J. Borysiuk, P. Prystawko, G. Kamler, T. Suski, and S. Porowski, "High power blue-violet InGaN laser diodes grown on bulk GaN substrates by plasma-assisted molecular beam epitaxy," Semicond. Sci. Technol., vol. 20, pp. 809-813, Aug. 2005.

[18] H. Y. Ryu, S. H. Kwon, Y. J. Lee, Y. H. Lee, and J. S. Kim, "Very-lowthreshold photonic band edge lasers from free-standing triangular photonic crystal slabs," Appl. Phys. Lett., vol. 80, pp. 3476-3478, May 2002.

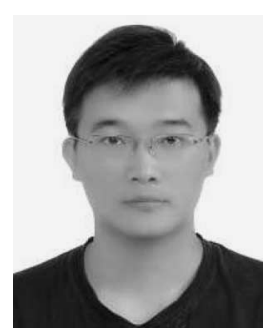

Shih Wei Chen received the B.S. degree in electrical engineering and the M.S. degree in optoelectronic sciences from the National Taiwan Ocean University (NTOU), Keelung, Taiwan, in 2004 and 2006, respectively. He is currently working toward the $\mathrm{Ph} . \mathrm{D}$. degree in electro-optical engineering at the National Chiao Tung University (NCTU), Hsinchu, Taiwan.

In 2006, he joined the Semiconductor Laser Technology Laboratory in NCTU. His current research interests are in the area of light emitting diode (LED), GaN vertical-cavity surface-emitting laser (VCSEL), high- $Q$ microcavity LED, and photonic crystal surface-emitting lasers (PCSEL) that can be applied to short-range fiber optical communication such as gigabit Ethernet and fiber channel under the instruction of Prof. Hao-Chung Kuo, Prof. Tien-Chang Lu, and Prof. Shing-Chung Wang.

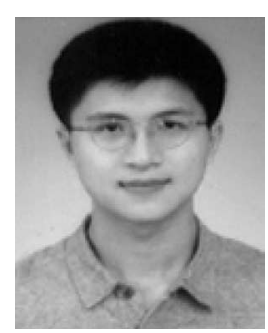

Tien Chang Lu received the B.S. degree in electrical engineering from the National Taiwan University, Taipei, Taiwan, in 1995, the M.S. degree in electrical engineering from the University of Southern California, Los Angeles, in 1998, and the Ph.D. degree in electrical engineering and computer science from the National Chiao Tung University, Hsinchu, Taiwan, in 2004.

In August 2005, he joined the Department of Photonics, National Chiao Tung University, as a faculty member. His current research interests include design, epitaxial growth, process and characterization of optoelectronic devices, such as Fabry-Perot-type semiconductor lasers, vertical-cavity surface-emitting lasers (VCSELs), resonant-cavity LEDs, microcavity, photonic crystal surfaceemitting lasers, wafer-fused flip-chip LEDs, solar cells, etc. He has authored or coauthored more than 100 international journal papers.

Prof. Lu was a recipient of the Exploration Research Award of the Pan Wen Yuan Foundation 2007 and the Excellent Young Electronic Engineer Award 2008.

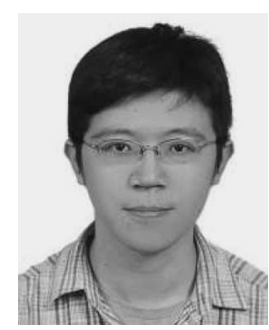

Tsung Ting Kao received the B.S. degree in electrophysics from the National Chiao Tung University (NCTU), Hsinchu, Taiwan, and the M.S. degree from the Department of Electro-Optical Engineering, NCTU, in 2006.

He was a member of the Semiconductor Laser Technology Laboratory under the supervision of Prof. Tien-Chang Lu, Prof. Hao-Chung Kuo, and Prof. Shing-Chung Wang in NCTU. He is currently a fulltime Research Assistant at NCTU. He is experienced in developing nitride-based optoelectronic devices and has numerous publications on GaN surface-emitting devices. His current research interests include GaN vertical-cavity surface-emitting laser (VCSEL), high- $Q$ microcavity LED, and photonic crystal surface-emitting lasers, etc. 